\title{
Upper-Body Contour Extraction Using Face and Body Shape Variance Information
}

\author{
Kazuki Hoshiai ${ }^{1}$, Shinya Fujie ${ }^{2}$, and Tetsunori Kobayashi ${ }^{1}$ \\ ${ }^{1}$ Department of Computer Science and Engineering, Waseda University, \\ Okubo 3-4-1, Shinjuku-ku, Tokyo 169-8555, Japan \\ hoshiai@pcl.cs.waseda.jp, koba@waseda.jp \\ ${ }^{2}$ Waseda Institute for Advanced Study, Waseda University, \\ Nishiwaseda 1-6-1, Shinjuku-ku, Tokyo 169-8050, Japan \\ fujie@pcl.cs.waseda.jp
}

\begin{abstract}
We propose a fitting method using a model that integrates face and body shape variance information for upper-body contour extraction. Accurate body-contour extraction is necessary for various applications, such as pose estimation, gesture recognition, and so on. In this study, we regard it as the shape model fitting problem. A model including shape variance information can fit to the contour robustly even in the noisy case. AAMs are one of these models and can fit to a face successfully. It needs appearance information for effective fitting, but it can not be used in our case because appearance of upper-body easily changes by clothes. Instead of intensity image, proposed method uses edge image as appearance information. However, discrimination between a true contour edge of upper-body and other edges is difficult. To solve this problem, we integrate shapes of upper-body and face. It is expected that this integrated model is more robust to edges in clutter background and various locations of the body than a body shape model using only body shape information. We conduct experiments and confirm improvement in accuracy by integration of face and body variance information.
\end{abstract}

Keywords: Contour extraction, Active Appearance Models, Active Body Shape Models, Active Integrated Shape Models.

\section{Introduction}

We propose a contour shape model integrating body shape variance information and face model of Active Appearance Models (AAMs) [12], and achieve accuracy improvement of upper-body contour extraction.

Extracting human contour with high accuracy is important to estimate positions of physical parts such as arm, head and so on. The most common approach to extract human contour is background subtraction [3]. A system such as a robot with active cameras can not utilize this approach which assumes fixed camera. There is "Snakes" [4] as another contour extraction method. It does not use prior knowledge about the contour shape. Owing to this, the model instance tends to converge on a wrong shape.

In this study, we regard body extraction problem as shape model fitting to a contour. We propose a new model and a novel fitting method using Inverse Compositional Image Alignment (ICIA) algorithm [25] of AAMs.

T. Wada, F. Huang, and S. Lin (Eds.): PSIVT 2009, LNCS 5414, pp. 862 873, 2009.

(C) Springer-Verlag Berlin Heidelberg 2009 


\section{Integration of Face and Body Shape Model}

We regard human contour extraction as shape model fitting to a body contour. In this study, we try to improve a model fitting accuracy, using the integrated model of face and body shape variance information.

This section describes fitting systems of face and body, and later describes a fitting system of integrated their systems.

\subsection{Face Model}

Active Shape Models (ASMs) [6] are a method of searching object contour using shape model. It has a habit of robustness to noise for learning the average shape and nonrigid deformation pattern using a set of training images preliminarily given the coordinates of feature points. For enormous amount of calculation, ASMs are extended to apply hierarchical approach using low-resolution images and motion prediction using Kalman filter for reducing iteration count of fitting [7]. One of the successful object detection and searching methods is AAMs. It has models constructed from both shape and appearance, and performs matching a normalized input image and a template image. AAMs allow real time tracking using ICIA algorithm as a model fitting algorithm.

ASMs and AAMs constrain deformation of the object shape which consists of a number of feature points by Principal Component Analysis (PCA). Their fitting algorithms have considerably difference between their models. After transferring each feature point, ASMs arrange the all points in accordance with PCA. On the other hand, AAMs transfer the general shape while maintaining consistency of the relation between the points, for solving the optimization problem of shape parameter based on eigen vector for fitting the object. As a result, AAMs can fit more accurately than ASMs. Furthermore, AAMs outperform ASMs about the convergence speed of fitting by ICIA algorithm.

The fitting process of AAMs is as follows. Firstly input images are transformed to template space using shape parameters. Secondly, the error of the translated image and the template image is calculated. Finally, the shape parameters, minimizing the error, are calculated using a face model. Model fitting to the face is performed using the shape parameters based on the calculation result. The fitting system of AAMs to face region is illustrated in Fig. 1(a).

\subsection{Body Model}

We apply AAMs to upper-body contour for fitting. When we use AAMs to face, a template image is a face image. A face includes universal information that does not depend on individual, so it can use intensity values as appearance information directly. On the other hand, it is difficult for upper-body model to use only intensity values inside its contour, because upper-body vision changes by clothes easily. Using the edge detected in boundary of human region and background region, we regard an edge image as appearance information to solve this problem.

The flow of fitting system is similar to a system of face except for regarding an edge image as an input image. The fitting system to upper-body contour is illustrated in Fig. 1(b). 


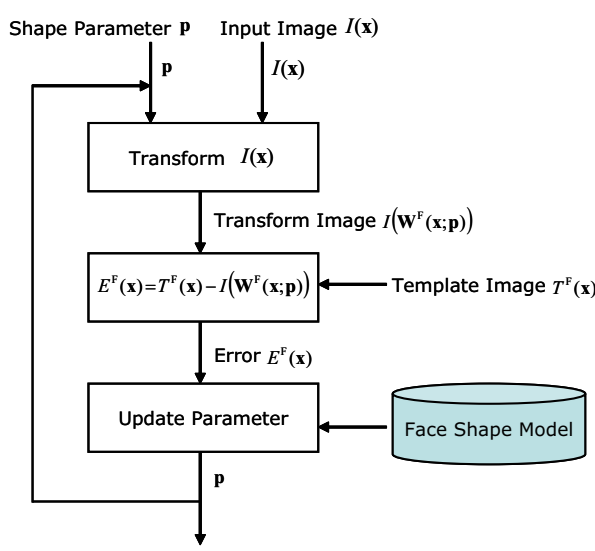

(a)

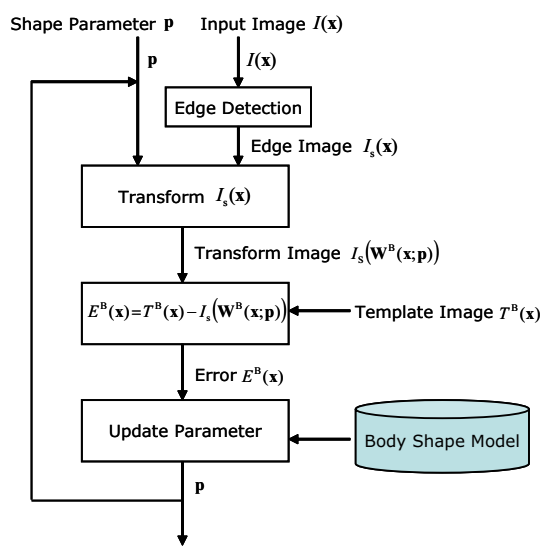

(b)

Fig. 1. Fitting algorithms for face shape model and upper-body shape model. (a) Fitting algorithm for face shape model, (b) Fitting algorithm for upper-body shape model.

\subsection{Integrated Model}

An edge image includes not only an upper-body contour edge of target person but also other edges. To distinguish an upper-body contour edge from other edges is difficult. Even in noisy environment, face model can be fitted because it does not depend on background. In this study, we integrate two models described in previous sections and

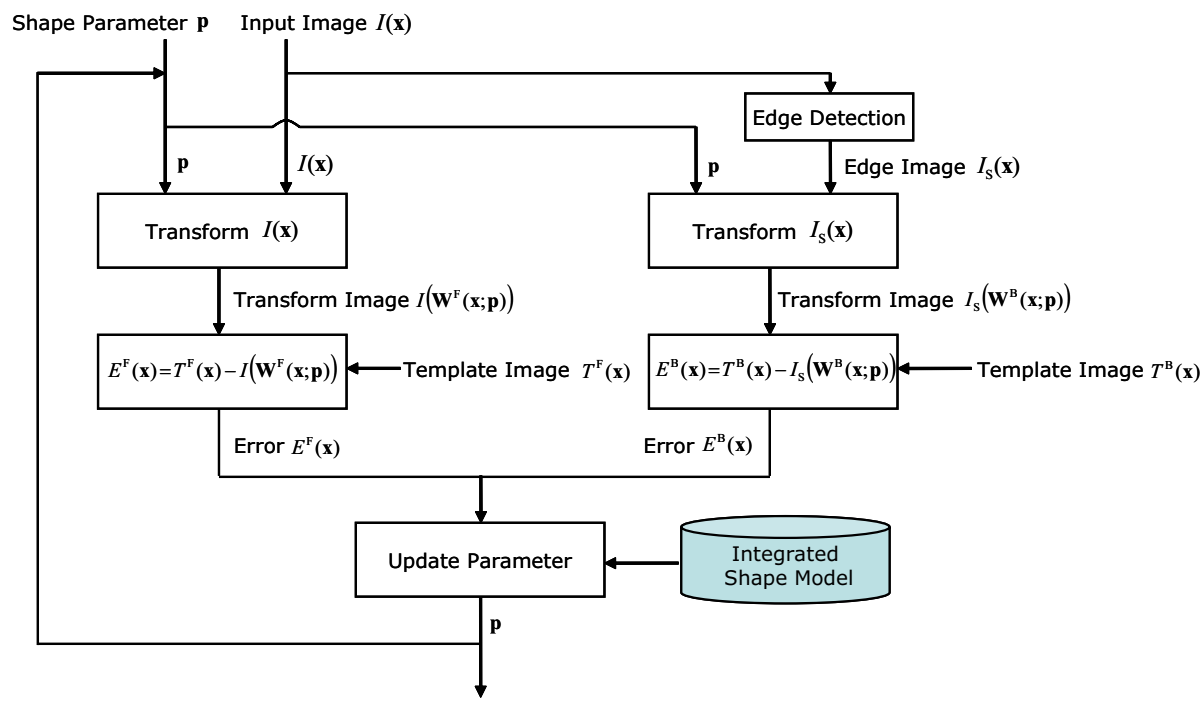

Fig. 2. Fitting algorithm for integrated model 
limit the search space for upper-body contour based on the position, direction and scale of face.

Procedure of fitting the model which is constructed from both face and body shape variance information is as follows. Firstly we perform transforming input image in face region, and edge image in upper-body contour, using integrated shape parameters of face and body shape variance. Secondly, the errors are calculated for face and body, separately. Finally, the shape parameters minimizing that errors are calculated by the integrated model. Model fitting to a face and a body contour is performed based on the shape parameters obtained by calculation result. The fitting system of integrated model is illustrated in Fig. 2 .

\section{Active Appearance Models}

We briefly overview the AAMs and their efficient fitting algorithm [12]. Then, we summarize the conditions which a model must satisfy, in order to apply that algorithm.

\subsection{Shape Model}

AAMs consist of two "active" models. First one is the shape model. A shape is defined as a set of $v$ vertices and lines connecting them, as shown in Fig. 3 (a). The shape vector $\mathbf{s}$ is represented as

$$
\mathbf{s}=\left[x_{1}, y_{1}, x_{2}, y_{2}, \cdots, x_{v}, y_{v}\right]^{T}
$$

where $x_{i}$ and $y_{i}$ represent $x$ and $y$ coordinate of $i$ th vertex respectively. PCA is applied to training data which are face images with hand-labeled feature points, then a shape is represented by linear sum of the average shape and the difference shapes,

$$
\mathbf{s}=\mathbf{s}_{0}+\sum_{i=1}^{n} p_{i} \mathbf{s}_{i}
$$

where $\mathbf{s}_{0}$ is the average shape and $\mathbf{s}_{i}$ is $i$ th principal component as a difference shape. Shape parameters are represented as the vector $\mathbf{p}=\left[p_{1}, p_{2}, \cdots, p_{n}\right]^{T}$. The shape of model instance is determined by the $\mathbf{p}$.

\subsection{Appearance Model}

Second model is an appearance model. Appearance model represents the variance of grayscale image in template space. Template space is defined as 2-D space constructed by the average shape. Applying PCA to the face images of training data represented in template space, an average appearance $A_{0}(\mathbf{x})$ and $m$ principal components $A_{i}(\mathbf{x})$ are calculated. $\mathbf{x}$ then describes pixels in template space. We regard the average appearance as the averaged face image, and the principal components as difference face image.

$$
A(\mathbf{x})=A_{0}(\mathbf{x})+\sum_{i=1}^{m} \lambda_{i} A_{i}(\mathbf{x})
$$




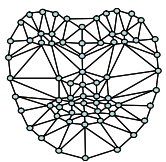

(a)

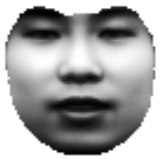

(b)

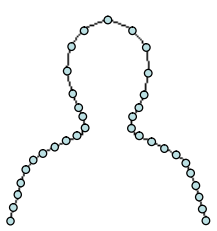

(c)

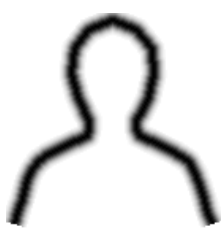

(d)

Fig. 3. Active Appearance Models and Active Body Shape Models. (a) Basic shape of AAMs $\mathbf{s}_{0}$, (b) Template image of AAMs $T(\mathbf{x})$, (c) Basic shape of ABSMs $\mathbf{s}_{0}^{\mathrm{B}}$, (d) Template image of ABSMs $T^{\mathrm{B}}(\mathbf{x})$.

Appearance parameters are represented as the vector $\boldsymbol{\lambda}=\left[\lambda_{1}, \lambda_{2}, \cdots, \lambda_{m}\right]^{T}$. The appearance of the model instance with respect to the face region is determined by the $\lambda$. The averaged face image $A_{0}(\mathbf{x})$ is regarded as the template image $T(\mathbf{x})$ shown in Fig. 3(b).

\subsection{Fitting Algorithm}

Fitting AAMs is regarded as minimizing the error between an input image and model instance. The error function is defined as

$$
E(\mathbf{x})=A_{0}(\mathbf{x})+\sum_{i=1}^{m} \lambda_{i} A_{i}(\mathbf{x})-I(W(\mathbf{x} ; \mathbf{p}))
$$

where $I(\mathbf{x})$ is an input image and $W(\mathbf{x} ; \mathbf{p})$ is a warp function warping points in template space to relative points in input image space. Note this warping function is determined by a shape parameter $\mathbf{p}$. For simplicity, we ignore appearance variation. Then, the target is to find a parameter $\mathbf{p}$ that minimizes the sum of square error function, that is

$$
\underset{\mathbf{p}}{\operatorname{argmin}} \sum_{\mathbf{x} \in \mathbf{s}_{0}}[T(\mathbf{x})-I(W(\mathbf{x} ; \mathbf{p}))]^{2} .
$$

Because, $I(\mathbf{x})$ is usually a non-linear function, it is difficult to calculate $\mathbf{p}$ directly. Then, AAMs use fitting algorithm named "Inverse Compositional Image Alignment (ICIA)." Small warp represented by $\Delta \mathrm{p}$ is introduced to template space,

$$
\underset{\Delta \mathbf{p}}{\operatorname{argmin}} \sum_{\mathbf{x} \in \mathbf{s}_{0}}[T(W(\mathbf{x} ; \Delta \mathbf{p}))-I(W(\mathbf{x} ; \mathbf{p}))]^{2} .
$$

$\Delta \mathbf{p}$ that minimizes the error function in context of given $\mathbf{p}$ is calculated.

\section{Active Body Shape Models}

We propose a fitting method of shape model using prior knowledge of contour variance information. We apply the framework of AAMs to body contour model in order to use prior knowledge. We call this model "Active Body Shape Models (ABSMs)." 


\subsection{Body Shape Model}

A body shape model is represented by the vector of arranged $x, y$ coordinate value of $u$ feature points.

$$
\mathbf{s}^{\mathrm{B}}=\left[x_{1}, y_{1}, x_{2}, y_{2}, \cdots, x_{u}, y_{u}\right]^{T}
$$

An average shape $\mathbf{s}^{\mathrm{B}}$ and $l$ principal components $\mathbf{s}_{i}^{\mathrm{B}}$ are calculated by PCA for all training data, which are similar to Eq. (2).

$$
\mathbf{s}^{\mathrm{B}}=\mathbf{s}_{0}^{\mathrm{B}}+\sum_{i=1}^{l} p_{i}^{\mathrm{B}} \mathbf{s}_{i}^{\mathrm{B}}
$$

Here, $p_{i}^{\mathrm{B}}$ describes the size of shape variance in terms of upper-body contour. In addition, shape parameters are represented as the vector $\mathbf{p}^{\mathrm{B}}=\left[p_{1}^{\mathrm{B}}, p_{2}^{\mathrm{B}}, \cdots, p_{l}^{\mathrm{B}}\right]^{T}$. The shape of the model instance with respect to the upper-body contour is determined by $\mathbf{p}^{\mathrm{B}}$.

\subsection{Applying ICIA Algorithm to ABSMs}

The differences of ABSMs from normal AAMs are (1) input and template images are edge images, and (2) shape is just a line not closed polygons. Former means that the gradient of template image cannot be calculated because edge image is a set of shape lines. It also causes a problem for the error function calculation, because edge image is very sparse, so the error value has low reliability. Latter means the piece-wise Affine transform cannot be used as the warp function directly. Against these problems, we redefine template image, input image, and warp function.

1) Template image: $T^{\mathrm{B}}(\mathbf{x})$

A line is constructed by connecting vertices in basic shape sequentially. We smooth this line to a constant distance and treat it as a template image. We show examples of a basic shape and a template image in Fig. 3 (c),(d). We fixed the extraction range above elbows, and defined the number of feature points was 35 , in this study.

2) Input image: $I_{\mathrm{S}}(\mathbf{x})$

The edge image is extracted by applying canny edge detector to original input image. Then this image is smoothed and the pixel values are normalized from 0 to 255 for the matching. We regard this image as new input image for ABSMs. An example of generation of an edge image is shown in Fig.4.

3) Warp function: $W^{\mathrm{B}}\left(\mathbf{x} ; \mathbf{p}^{\mathrm{B}}\right)$

Template space is regarded as belt-like space around a line linking the feature points. This belt-like space is constructed by the set of quadrangular area. We can transform each quadrangular area in template space to input image space. The dividing boundaries are bisectors of angles between two lines through an objective point and two points next to that point. The warp function mapping from input image space to template space is piecewise bilinear warp using bilinear transform [8] for each quadrangle area. We consider a case of projection about four vertices of a certain quadrangle area in template 

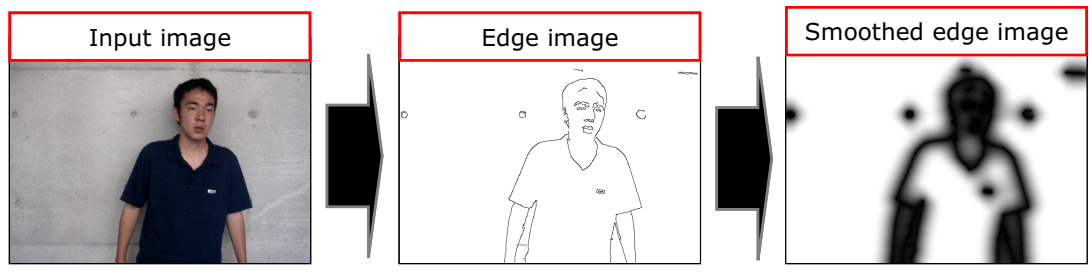

Fig. 4. Process of forming the smoothed edge image used for matching with the template image

space to $\left(x_{00}, y_{00}\right),\left(x_{10}, y_{10}\right),\left(x_{01}, y_{01}\right),\left(x_{11}, y_{11}\right)$ with the shape parameter $\mathbf{p}^{\mathrm{B}}$. When each pixel is denoted by $\mathbf{x}=[x, y]^{T}$, the warp function is described as follows.

$$
\begin{aligned}
W^{\mathrm{B}}\left(\mathbf{x} ; \mathbf{p}^{\mathrm{B}}\right)= & (1-\alpha)(1-\beta)\left[x_{00}, y_{00}\right]^{T}+\alpha(1-\beta)\left[x_{10}, y_{10}\right]^{T} \\
& +(1-\alpha) \beta\left[x_{01}, y_{01}\right]^{T}+\alpha \beta\left[x_{11}, y_{11}\right]^{T}
\end{aligned}
$$

$\alpha$ and $\beta$ are transform coefficients of bilinear transform from template space to square space.

\section{Integration of ABSMs and AFAMs}

The upper-body extraction using only edge information is difficult, when there are many edges in background as well as the upper-body contour. In this section, we describe how we integrate two models to tackle this problem.

In following part, we call normal AAMs for face as "Active Face Appearance Models (AFAMs)," and attach $\mathrm{F}$ as superscript to parameters and model specific functions for AFAMs.

\subsection{Integration of Shape Models}

The new shape model is a model obtained by integrating two models, the body shape model of ABSMs and the face shape model of AFAMs. We call this model "integrated shape model."

$$
\mathbf{s}=\left[x_{1}, y_{1}, x_{2}, y_{2}, \cdots, x_{v+u}, y_{v+u}\right]^{T}
$$

An average shape $\mathbf{s}_{0}$ and $k$ principal components $\mathbf{s}_{i}$ are calculated by PCA for all training data.

$$
\mathbf{s}=\mathbf{s}_{0}+\sum_{i=1}^{k} p_{i} \mathbf{s}_{i}
$$

In addition, shape parameters are represented as the vector $\mathbf{p}=\left[p_{1}, p_{2}, \cdots, p_{k}\right]^{T}$. The shape of the model instance with respect to both the upper-body contour and the face region is specified by the $\mathbf{p}$. An appearance model is constructed for only face appearance model of AFAMs. We call the model consisting of integrated shape model and face shape model and the fitting algorithm of it "Active Integrated Shape Models (AISMs)." 


\subsection{Fitting Algorithm}

The evaluation function of fitting AISMs is defined as weighted sum of error for upperbody and error of face.

$$
\begin{array}{r}
\underset{\Delta \mathbf{p}}{\operatorname{argmin}}\left((1-w) \sum_{\mathbf{x} \in \mathbf{s}_{0}^{\mathrm{B}}}\left[T^{\mathrm{B}}\left(W^{\mathrm{B}}(\mathbf{x} ; \Delta \mathbf{p})\right)-I_{\mathrm{s}}\left(W^{\mathrm{B}}(\mathbf{x} ; \mathbf{p})\right)\right]^{2}\right. \\
\left.+w \sum_{\mathbf{x} \in \mathbf{s}_{0}^{\mathrm{F}}}\left[T^{\mathrm{F}}\left(W^{\mathrm{F}}(\mathbf{x} ; \Delta \mathbf{p})\right)-I\left(W^{\mathrm{F}}(\mathbf{x} ; \mathbf{p})\right)\right]^{2}\right)
\end{array}
$$

The range of weight is $0<w<1$. This weight represents ratio of influence by face information and body information. In this study, we empirically determined $w=0.4$. We take the Taylor series expansion of $T^{\mathrm{B}}\left(W^{\mathrm{B}}(\mathbf{x} ; \Delta \mathbf{p})\right)$ and $T^{\mathrm{F}}\left(W^{\mathrm{F}}(\mathbf{x} ; \Delta \mathbf{p})\right)$ in Eq. (12).

$$
\begin{aligned}
(1-w) & \sum_{\mathbf{x} \in \mathbf{s}_{0}^{\mathrm{B}}}\left[T^{\mathrm{B}}(\mathbf{x})+\nabla T^{\mathrm{B}}(\mathbf{x}) \frac{\partial W^{\mathrm{B}}}{\partial \mathbf{p}} \Delta \mathbf{p}-I_{\mathrm{s}}\left(W^{\mathrm{B}}(\mathbf{x} ; \mathbf{p})\right)\right]^{2} \\
+w & \sum_{\mathbf{x} \in \mathbf{s}_{0}^{\mathrm{F}}}\left[T^{\mathrm{F}}(\mathbf{x})+\nabla T^{\mathrm{F}}(\mathbf{x}) \frac{\partial W^{\mathrm{F}}}{\partial \mathbf{p}} \Delta \mathbf{p}-I\left(W^{\mathrm{F}}(\mathbf{x} ; \mathbf{p})\right)\right]^{2}
\end{aligned}
$$

$\Delta$ p minimizing Eq. (13) is computed.

$$
\begin{array}{r}
\Delta \mathbf{p}=\mathbf{H}^{-1}\left((1-w) \sum_{\mathbf{x} \in \mathbf{s}_{0}^{\mathrm{B}}}\left[\nabla T^{\mathrm{B}}(\mathbf{x}) \frac{\partial W^{\mathrm{B}}}{\partial \mathbf{p}}\right]\left[I_{\mathrm{s}}\left(W^{\mathrm{B}}(\mathbf{x} ; \mathbf{p})\right)-T^{\mathrm{B}}(\mathbf{x})\right)\right] \\
\left.\left.+w \sum_{\mathbf{x} \in \mathbf{s}_{0}^{\mathrm{F}}}\left[\nabla T^{\mathrm{F}}(\mathbf{x}) \frac{\partial W^{\mathrm{F}}}{\partial \mathbf{p}}\right]\left[I\left(W^{\mathrm{F}}(\mathbf{x} ; \mathbf{p})\right)-T^{\mathrm{F}}(\mathbf{x})\right)\right]\right)
\end{array}
$$

Then, we denote hessian matrix $\mathbf{H}$ as follows.

$$
\begin{array}{r}
\mathbf{H}=(1-w) \sum_{\mathbf{x} \in \mathbf{s}_{0}^{\mathrm{B}}}\left[\nabla T^{\mathrm{B}}(\mathbf{x}) \frac{\partial W^{\mathrm{B}}}{\partial \mathbf{p}}\right]^{T}\left[\nabla T^{\mathrm{B}}(\mathbf{x}) \frac{\partial W^{\mathrm{B}}}{\partial \mathbf{p}}\right] \\
+w \sum_{\mathbf{x} \in \mathbf{s}_{0}^{\mathrm{F}}}\left[\nabla T^{\mathrm{F}}(\mathbf{x}) \frac{\partial W^{\mathrm{F}}}{\partial \mathbf{p}}\right]^{T}\left[\nabla T^{\mathrm{F}}(\mathbf{x}) \frac{\partial W^{\mathrm{F}}}{\partial \mathbf{p}}\right]
\end{array}
$$

$\Delta \mathrm{p}$ minimizing Eq.(12) is calculated analytically by Eq.(14). $\mathrm{p}$ can be updated sequentially from obtained $\Delta \mathbf{p}$ and current $\mathbf{p}$. The optimal shape parameter is provided by $\Delta \mathbf{p}$ sequentially, and fitting to the body contour and the face is performed simultaneously.

\section{Experiments}

\subsection{ABSMs Experiment}

We evaluated the fitting to upper-body contour. We conducted comparative experiments for evaluating accuracy with proposed ABSMs of the modeling method described in 4 and ICIA algorithm described in 4.2 
Setup. We performed data collection to prepare test data for the evaluation and learning data to construct the body shape model. Subjects were 15 students in our laboratory. The collected data included four kinds of patterns.

1. facing on the front

2. facing sideways a little

3. inclining to front and back

4. inclining to right and left

The subjects performed the indicated movement of each pattern. We collected $200 \mathrm{im}-$ age data, capturing the video corresponding to each pattern. The examples of experiment data are shown in Fig. 5.

We computed the error between fitting result and labeled data. We regarded the distance from the correct feature point to a nearest pixel on the fitting result as the error. The image data for experiment were 200 images described in 6.2. The data were divided into four sets, and then one set consists of 50 images. Three sets including 150 images were learning data, and one set was test data. We performed experiments with four combinations. We utilized Snakes which is widely used for the contour extraction as comparison method of ABSMs. Drawing a line by connecting the start point and the end point of feature points, we generated the edge, and artificially made closed region. We performed the model fitting to the closed region of upper-body contour. We set the same shape of initial model instance. These initial model instances were randomly determined as slightly larger than hand labeled correct shapes. We set the distance $d$, of which there are ten kinds from 1 to 10 , from the model instances. We prepared one hundred combinations of the points which draw apart from the three correct feature points, and measure the error for each combination.

Results and discussion. We calculated the error per one data of Snakes and proposed ABSMs. Note that the unconverged data were eliminated for this evaluation. We calculated the mean of the errors of all test data. They are 475.0 for Snakes and 378.5 for ABSMs. This result shows that ABSMs is more accurate than the traditional method.

The model instance of Snakes converged inside the closed region consisting of the upper-body contour, when the closed region was broken by lacking the edge over the upper-body contour. In contrast, ABSMs fitted the contour successfully even in such a case, because the model instance keeps the shape consistency for the body shape model.

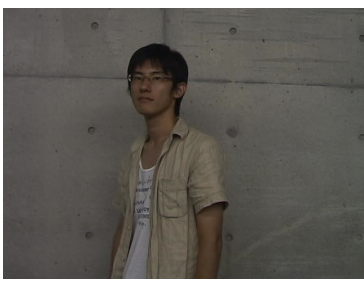

(a)

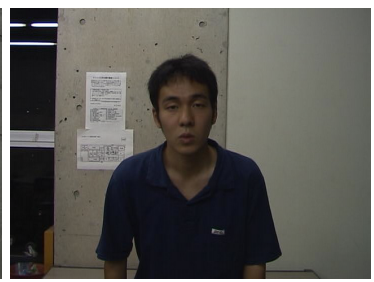

(b)

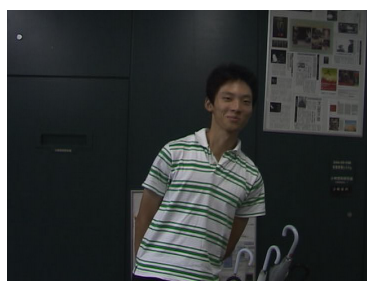

(c)

Fig. 5. The example of experiment data: (a) facing sideway, (b) inclining to front, (c) inclining to left 


\subsection{AISMs Experiment}

We conducted an experiment with ABSMs and AISMs to measure the model fitting accuracy to upper-body contour.

Setup. We performed data collection to prepare test data for the evaluation and learning data to construct the models. Subjects were 5 students in our laboratory. The collecting data included three kinds of pattern.

1. facing on the front

2. facing sideway a little

3. inclining to right

We gave instruction to the subjects to pose each pattern. We captured the image at random from the video and collected in total 500 images which includes 100 images per subject. We labeled feature points by hand to a face and a body contour on the captured images, and these points coordinate were used as correct label representing shape.

In this study, we used total 103 of the feature points which consist of 68 face points and 35 upper-body contour points. We show an labeled image and the feature point location in Fig.6

We used 100 images of one subject as test data and the other data as learning data, and performed 5-fold cross validation. We used the images of training data sets and flipped images of them to construct the models. 481 images succeeded in face detection. The shape parameter of ABSMs converged for 440 images. The shape parameter of AISMs converged for 399 images. The shape parameter of both ABSMs and AISMs converged for 393 image. The face position was determined automatically by the face detector [9], and the position of initial model instance was determined by the face position around the body. We updated the shape parameter until it converges. When the parameter converges, we measured the distance error per point between the model instance of feature points and the hand-labeled correct feature points. We counted the error of the data which converge both of ABSMs and AISMs, and then we calculated the averaged error per feature point.

Results and discussion. The averaged errors per feature point for ABSMs and AISMs were 9.9 and 8.7 respectively. Fig. 7 shows the cumulative frequency distribution of

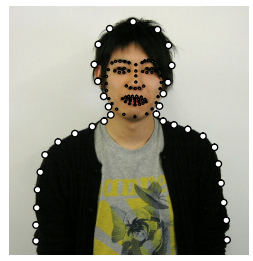

(a)

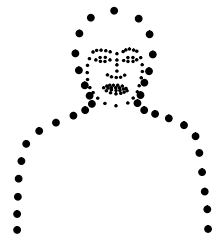

(b)

Fig. 6. The example of labeled image: big points are feature points of upper-body, and small points are feature points of face region, (a) Labeled image, (b) Feature points location 


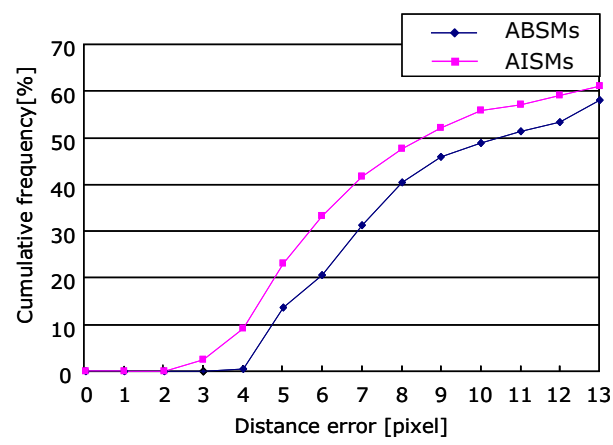

Fig. 7. Cumulative frequency distribution of the distance error per feature point for ABSMs and AISMs

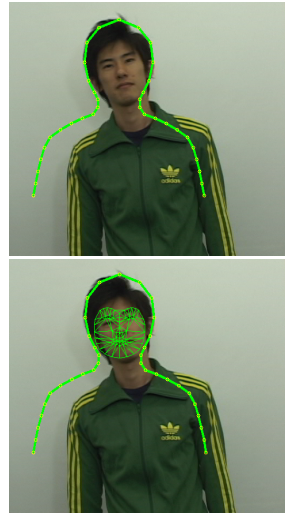

(a) Iteration 1

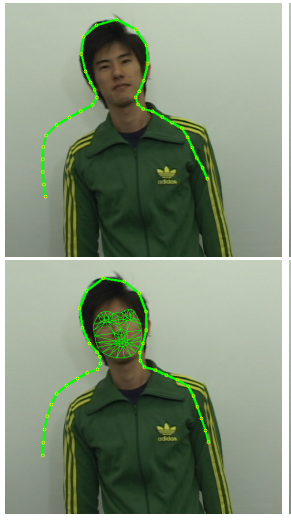

(b) Iteration 50

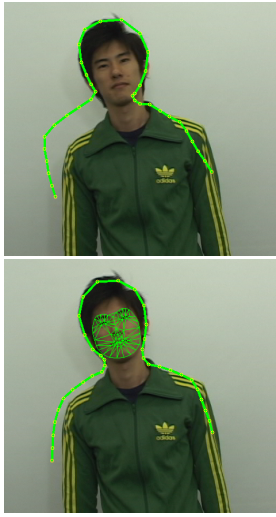

(c) Iteration 100

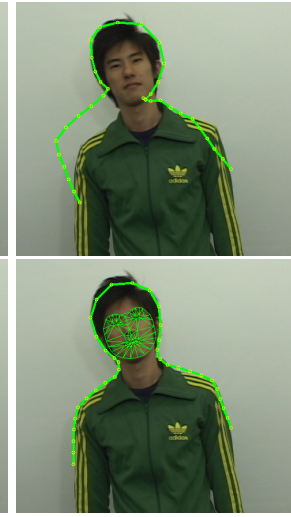

(d) Iteration 150

Fig. 8. Process of fitting convergence, the upper row:ABSMs, and the lower row:AISMs (Iteration count $1,50,100,150)$

the distance error per feature point. This result concludes that AISMs often converge nearer than ABSMs. We confirmed that the integration of the face information made the error reduced. The precision of the model fitting are improved by the integration of ABSMs and AFAMs. The fitting process of the model instance is shown in Fig. 8 The round points show feature points, and the line of the model instance shows the result of upper-body extraction.

We discuss the experiment result of Fig. 8 8 , The position of the initial model instance was determined by the face region. Thereby, the upper-body was considerably away from correct position with respect to this sample. In consequence, the model instance did not fit to the contour by ABSMs. However, AISMs succeeded in the body part of the model fitting to the contour while the face part of the model instance turned around to the correct position by using face information. The upper-body contour and face shape variance constrained each other by integration of body and face shape, while fitting to 
the face using the input image, the model instance fits to the contour along the position, direction, and scale of face. Therefore the model instance fitted the upper-body contour.

\section{Conclusions}

For upper-body contour extraction, we proposed ABSMs as the fitting method using ICIA algorithm. ABSMs has an upper-body contour shape model that represents shape variance information. We confirmed that fitting precision of ABSMs was high in comparison with the traditional method to the upper-body extraction by the experiment. Furthermore, we proposed AISMs as the fitting method that integrated AFAMs and ABSMs. We introduced weight for a face and physical error function in AISMs and defined new evaluation function. Proposed method can estimate the most suitable shape parameter using ICIA algorithm for this evaluation function. We evaluated the fitting precision for ABSMs and AISMs. As a result, high fitting precision of AISMs was achieved in comparison with ABSMs and we showed the effectiveness of using face information for the issue of upper-body contour extraction.

In this study, we used a fixed weight for the error function of face and the upper-body contour extraction. In future, we are going to use a dynamic weight obeying a reliability with an upper-body contour extraction and a face information. This reliability can be calculated with the complexity of the edge by the background. Moreover we aim at precision improvement by taking in the appearance information of clothes and using much appearance information.

\section{Acknowledgment}

This work was partly supported by New Energy and Industrial Technology Development Organization (NEDO), Japan.

\section{References}

1. Cootes, T.F., Edwards, G.J., Taylor, C.J.: Active appearance models. IEEE Trans. on Pattern Analysis and Machine Intelligence 23(6), 681-685 (2001)

2. Matthews, I., Baker, S.: Active appearance models revisited. International Journal of Computer Vision 60(2), 1405-1573 (2004)

3. Li, L., Huang, W., Gu, I.Y., Tian, Q.: Statistical modeling of complex backgrounds for foreground object detection. IEEE Trans. on Image Processing 13(11), 1495-1472 (2004)

4. Kass, M., Witkin, A., Terzopoulos, D.: Snakes: Active contour models. International Journal of Computer Vision 1(4), 321-331 (1988)

5. Baker, S., Matthews, I.: Lucas-kanade 20 years on: A unifying framework. International Journal of Computer Vision 56(3), 221-255 (2004)

6. Cootes, T.F., Taylor, C.J., Cooper, D.H., Graham, J.: Active shape models-their training and application. Computer Vision and Image Understanding 61(1), 38-59 (1995)

7. Lee, S.W., Kang, J., Shin, J., Paik, J.: Hierarchical active shape model with motion prediction for real-time tracking of non-rigid objects. IET Computer Vision 1(1), 17-24 (2007)

8. Heckbert, P.S.: Fundamentals of texture mapping and image warping. Master's thesis, University of California (1989)

9. Viola, P., Jones, M.J.: Rapid object detection using a boosted cascade of simple features. International Journal of Computer Vision 57(2), 137-154 (2004) 